\title{
DECOMPOSITION METHOD FOR NONSMOOTH CONVEX VARIATIONAL SIGNAL RECOVERY
}

\author{
Heinz H. Bauschke, Patrick L. Combettes, ${ }^{2}$ and Jean-Christophe Pesquet ${ }^{3}$ \\ ${ }^{1}$ Mathematics, University of British Columbia Okanagan, Kelowna, BC V1V 1V7, Canada \\ ${ }^{2}$ Laboratoire Jacques-Louis Lions, Université Pierre et Marie Curie - Paris 6, 75005 Paris, France \\ ${ }^{3}$ Institut Gaspard Monge and UMR-CNRS 8049, Université de Marne la Vallée, 77454 Marne la Vallée Cedex 2, France
}

\begin{abstract}
Under consideration is the large body of signal recovery problems that can be formulated as the problem of minimizing the sum of two proper lower semicontinous convex (not necessarily smooth) functions in a real Hilbert space. A general analysis of this problem is given and a decomposition method is proposed to solve it. The convergence of the method, which is based on an extension of the standard Douglas-Rachford algorithm for monotone operators, is established under genral conditions. Various signal recovery applications are discussed and numerical results are provided.
\end{abstract}

\section{INTRODUCTION}

A wide array of methodological approaches have been proposed to solve signal recovery problems based on various physical, statistical, and numerical considerations, as well as on certain heuristic beliefs. Mathematically, though, the vast majority of signal recovery problems are posed as optimization problems and typically solved on a case-by-case basis by an ad hoc numerical algorithm. In [3], it was shown that a number of apparently unrelated problems fitted the following variational format in a real Hilbert space $\mathcal{H}$.

Problem 1 Let $\left.\left.f_{1}: \mathcal{H} \rightarrow\right]-\infty,+\infty\right]$ and $f_{2}: \mathcal{H} \rightarrow \mathbb{R}$ be two proper lower semicontinuous convex functions such that $f_{2}$ is differentiable on $\mathcal{H}$ with a $1 / \beta$-Lipschitz continuous gradient, for some $\beta \in] 0,+\infty[$. The objective is to minimize $f_{1}+f_{2}$ over $\mathcal{H}$.

Problem 1 was shown to cover a variety of signal recovery formulations, including constrained least-squares problems, multiresolution sparse regularization problems, Fourier regularization problems, geometry/texture image decomposition problems, hard-constrained inconsistent feasibility problems, split feasibility problems, as well as certain maximum a posteriori problems [3]. Investigating this generic formulation therefore made it possible to derive existence, uniqueness, characterization, and stability results in a unified and standardized fashion for a large class of problems. Moreover, a relaxed forward-backward algorithm was proposed to solve Problem 1 and shown to capture, extend, and provide a simplified analysis for a variety of existing iterative methods, such as the projected Landweber method, alternating projections methods, or the iterative thresholding method recently proposed in [4] (see [3] for details).

Despite its relatively broad scope, Problem 1 fails to cover the important situations in which $f_{2}$ is differentiable with a non-Lipschitz gradient, or not finite everywhere, or, more importantly, when it is not differentiable at all. The latter situation includes for instance the problem of minimizing the total variation of a signal over a convex set, the problem of minimizing the sum of two set-distances, problems in which both functions are maxima of convex functions, or Tykhonov-like problems with $L^{1}$ norms. The objectives of the present paper are to extend Problem 1 by relaxing the assumptions on $f_{2}$ to a mere standard qualification condition, to analyze the properties of the resulting nonsmooth convex optimization problem, and to propose an iterative decomposition method to solve it based on recent developments [1] on the Douglas-Rachford method for monotone operator splitting [7]. In Section 3, we define our notation and provide the necessary mathematical background. The recovery problem is formulated, analyzed, and illustrated in Section 2. In Section 4, an algorithm is proposed to solve it. Finally, numerical applications to waveletbased signal recovery are presented in Section 5.

\section{NOTATION AND THEORETICAL TOOLS}

Throughout this paper, $\mathcal{H}$ is a real Hilbert space with scalar product $\langle\cdot \mid \cdot\rangle$, norm $\|\cdot\|$, and distance $d$.

\subsection{Convex analysis [9]}

The indicator function of a subset $C$ of $\mathcal{H}$ is

$$
\iota_{C}: x \mapsto \begin{cases}0, & \text { if } x \in C ; \\ +\infty, & \text { if } x \notin C,\end{cases}
$$


and the distance from a point $x \in \mathcal{H}$ to $C$ is $d_{C}(x)=$ $\inf \|x-C\|$; if $C$ is also closed and convex then, for every $x \in \mathcal{H}$, there exists a unique point $P_{C} x \in C$ such that $\left\|x-P_{C} x\right\|=d_{C}(x) ; P_{C} x$ is the projection of $x$ onto $C$.

The domain of a function $f: \mathcal{H} \rightarrow]-\infty,+\infty]$ is $\operatorname{dom} f=\{x \in \mathcal{H} \mid f(x)<+\infty\} . \quad \Gamma_{0}(\mathcal{H})$ is the class of all lower semicontinuous convex functions from $\mathcal{H}$ to ]$-\infty,+\infty]$ which are proper in the sense that their domain is nonempty. Now let $f \in \Gamma_{0}(\mathcal{H})$. The conjugate of $f$ is the function $f^{*} \in \Gamma_{0}(\mathcal{H})$ defined by

$$
(\forall u \in \mathcal{H}) f^{*}(u)=\sup _{x \in \mathcal{H}}\langle x \mid u\rangle-f(x)
$$

and $f^{* *}=f$. The subdifferential of $f$ at $x \in \mathcal{H}$ is the set $\partial f(x)=\{u \in \mathcal{H} \mid(\forall y \in \mathcal{H})\langle y-x \mid u\rangle+f(x) \leq f(y)\}$.

If $f$ is (Gâteaux) differentiable at $x$ with gradient $\nabla f(x)$, then $\partial f(x)=\{\nabla f(x)\}$.

Lemma 2 Let $f_{1}$ and $f_{2}$ be two functions in $\Gamma_{0}(\mathcal{H})$ such that

$$
\bigcup_{\gamma>0}\left\{\gamma\left(x_{1}-x_{2}\right) \mid x_{1} \in \operatorname{dom} f_{1}, x_{2} \in \operatorname{dom} f_{2}\right\}
$$

is a closed vector subspace,

and let $x \in \mathcal{H}$. Then $x$ minimizes $f_{1}+f_{2}$ if and only if $0 \in \partial f_{1}(x)+\partial f_{2}(x)$.

Remark 3 Condition (4) is satisfied in each of the following cases.

(i) $f_{1}$ or $f_{2}$ is finite.

(ii) $\operatorname{dom} f_{1} \cap \operatorname{int} \operatorname{dom} f_{2} \neq \varnothing$ or dom $f_{2} \cap \operatorname{int} \operatorname{dom} f_{1} \neq \varnothing$.

(iii) $\operatorname{dim} \mathcal{H}<+\infty$ and the relative interior of $\operatorname{dom} f_{1}$ intersects with that of $\operatorname{dom} f_{2}$.

\subsection{Proximity operators [3]}

Let $f \in \Gamma_{0}(\mathcal{H})$. Then, for every $x \in \mathcal{H}$, the function $y \mapsto$ $f(y)+\|x-y\|^{2} / 2$ admits a unique minimizer denoted by $\operatorname{prox}_{f} x$. The operator

$$
\operatorname{prox}_{f}: \mathcal{H} \rightarrow \mathcal{H}: x \mapsto \underset{y \in \mathcal{H}}{\arg \min } f(y)+\frac{1}{2}\|x-y\|^{2}
$$

is the proximity operator of $f$.

Lemma 4 Let $f \in \Gamma_{0}(\mathcal{H})$. Then

(i) $(\forall \gamma \in] 0,+\infty[)(\forall x \in \mathcal{H})$

$$
x=\operatorname{prox}_{\gamma f} x+\gamma \operatorname{prox}_{f * / \gamma}(x / \gamma) .
$$

(ii) $(\forall x \in \mathcal{H})(\forall y \in \mathcal{H})\left\|\operatorname{prox}_{f} x-\operatorname{prox}_{f} y\right\|^{2}$

$$
\leq\|x-y\|^{2}-\left\|\operatorname{prox}_{f^{*}} x-\operatorname{prox}_{f^{*}} y\right\|^{2} .
$$

(iii) If $f$ is even, then $\operatorname{prox}_{f}$ is odd.

\subsection{Examples of proximity operators [3]}

Example 5 Set $f=\iota_{C}$, where $C$ is a nonempty closed convex subset of $\mathcal{H}$. Then $\operatorname{prox}_{f}=P_{C}$. Hence, proximity operators generalize the notion of a projection operator.

Example 6 Let $C$ be a nonempty closed convex subset of $\mathcal{H}$, let $\gamma \in] 0,+\infty[$, and let $x \in \mathcal{H}$. Then

$$
\operatorname{prox}_{\gamma d_{C}} x= \begin{cases}x+\frac{\gamma}{d_{C}(x)}\left(P_{C} x-x\right), & \text { if } d_{C}(x)>\gamma ; \\ P_{C} x, & \text { if } d_{C}(x) \leq \gamma\end{cases}
$$

Example 7 If $\mathcal{H}=\mathbb{R}$ and $C=\{0\}$ in Example 6, we obtain the soft-thresholding operation

$$
\operatorname{prox}_{\gamma|\cdot|} x=\operatorname{sgn}(x) \max \{|x|-\gamma, 0\} \text {. }
$$

Example 8 Let $\left(e_{k}\right)_{k \in \mathbb{N}}$ be an orthonormal basis of $\mathcal{H}$, let $\left(\phi_{k}\right)_{k \in \mathbb{N}}$ be functions in $\Gamma_{0}(\mathbb{R})$ such that

$$
(\forall k \in \mathbb{N}) \phi_{k} \geq 0 \quad \text { and } \quad \phi_{k}(0)=0,
$$

let $f: \mathcal{H} \rightarrow]-\infty,+\infty]: x \mapsto \sum_{k \in \mathbb{N}} \phi_{k}\left(\left\langle x \mid e_{k}\right\rangle\right)$, and let $x \in \mathcal{H}$. Then $\psi \in \Gamma_{0}(\mathcal{H})$ and $\operatorname{prox}_{f} x=\sum_{k \in \mathbb{N}} \pi_{k} e_{k}$, where $\pi_{k}=\operatorname{prox}_{\phi_{k}}\left\langle x \mid e_{k}\right\rangle$ is the unique solution to the inclusion $\left\langle x \mid e_{k}\right\rangle-\pi_{k} \in \partial \phi_{k}\left(\pi_{k}\right)$.

Example 9 Let $K$ be a (finite of infinite) subset of $\mathbb{N}$, let $\left(e_{k}\right)_{k \in K}$ be an orthonormal basis of $\mathcal{H}$, let $\left(p_{k}\right)_{k \in K}$ be a sequence in $\left[1,+\infty\left[\right.\right.$, let $\left(\omega_{k}\right)_{k \in K}$ be a sequence in ] $0,+\infty[$, let $f: \mathcal{H} \rightarrow]-\infty,+\infty]: x \mapsto \sum_{k \in K} \omega_{k}\left|\left\langle x \mid e_{k}\right\rangle\right|^{p_{k}}$, and let $x \in \mathcal{H}$. Then $\operatorname{prox}_{f} x=\sum_{k \in K} \pi_{k} e_{k}$ where, for every $k \in K, \pi_{k}$ is the unique solution to the equation

$$
p_{k} \omega_{k}\left|\pi_{k}\right|^{p_{k}-1} \operatorname{sgn}\left(\pi_{k}\right)+\pi_{k}-\xi_{k}=0,
$$

where $\xi_{k}=\left\langle x \mid e_{k}\right\rangle$; in particular, $\pi_{k}$ is given by

$$
\begin{cases}\operatorname{sgn}\left(\xi_{k}\right) \max \left\{\left|\xi_{k}\right|-\omega_{k}, 0\right\}, & \text { if } p_{k}=1 \\ \xi_{k} /\left(1+2 \omega_{k}\right), & \text { if } p_{k}=2 \\ \operatorname{sgn}\left(\xi_{k}\right)\left(\left|\xi_{k}\right|+\frac{9}{8} \omega_{k}^{2}\left(1-\sqrt{1+\frac{16\left|\xi_{k}\right|}{9 \omega_{k}^{2}}}\right)\right) & \text { if } p_{k}=\frac{3}{2} \\ \operatorname{sgn}\left(\xi_{k}\right) \frac{\sqrt{1+12 \omega_{k}\left|\xi_{k}\right|}-1}{6 \omega_{k}}, & \text { if } p_{k}=3\end{cases}
$$

Example 10 Let $\mathcal{H}=\mathbb{R}^{m}$, let $\left.\gamma \in\right] 0,+\infty[$, and let $f$ be the Burg entropy, i.e., $f: x=\left(\xi_{k}\right)_{1 \leq k \leq m} \mapsto \sum_{k=1}^{m} \phi\left(\xi_{k}\right)$, where

$$
\phi: \xi \mapsto \begin{cases}-\ln (\xi), & \text { if } \xi>0 ; \\ +\infty, & \text { if } \xi \leq 0 .\end{cases}
$$

Then

$$
\left(\forall x \in \mathbb{R}^{m}\right) \operatorname{prox}_{\gamma f} x=\frac{1}{2}\left(\xi_{k}+\sqrt{\xi_{k}^{2}+4 \gamma^{2}}\right)_{1 \leq k \leq m} .
$$




\section{PROBLEM FORMULATION}

Problem 11 Let $f_{1}$ and $f_{2}$ be two functions in $\Gamma_{0}(\mathcal{H})$ which satisfy (4). The objective is to

$$
\underset{x \in \mathcal{H}}{\operatorname{minimize}} f_{1}(x)+f_{2}(x) .
$$

\section{Proposition 12}

(i) Existence: Problem 11 possesses at least one solution if $\lim _{\|x\| \rightarrow+\infty} f_{1}(x)+f_{2}(x)=+\infty$.

(ii) Uniqueness: Problem 11 possesses at most one solution if $f_{1}+f_{2}$ is strictly convex, as is the case when $f_{1}$ or $f_{2}$ is strictly convex.

(iii) Characterization: Let $x \in \mathcal{H}$ and $\gamma \in] 0,+\infty[$, and set $\operatorname{rprox}_{f}=2 \operatorname{prox}_{f}-$ Id. Then the following statements are equivalent.

(a) $x$ solves Problem 11.

(b) $x=\operatorname{prox}_{\gamma f_{2}} y$, where $y=\operatorname{rprox}_{\gamma f_{1}} \operatorname{rprox}_{\gamma f_{2}} y$.

Proof. (i)\&(ii): [3, Proposition 3.1]. (iii): Combine [1, Lemma 2.6(iii)] and Lemma 2.

Let us note that the only restriction imposed in Problem 11 is the relatively mild qualification condition (4). It therefore follows from Remark 3(i) that Problem 11 subsumes Problem 1. Therefore, all the examples of signal recovery problems discused in [3] are covered by Problem 11. We now list a few concrete scenarios which are covered by Problem 11 but not by Problem 1 .

Example $13 \mathcal{H}$ is either or $\mathbb{R}^{N}$ or $H^{1}(\Omega)$, where $\Omega$ is an open bounded domain of $\mathbb{R}^{m}, f_{1}$ is the $\mathrm{L}^{1}$ norm or the indicator function of a nonempty closed convex set, and $f_{2}$ is the total variation. This follows from [2, Proposition 1].

Example $14 C_{1}$ and $C_{2}$ are two nonempty closed convex sets, $f_{1}=d_{C_{1}}$, and $f_{2}=d_{C_{2}}$. Problem 11 is then an extension of the standard convex feasiblity problem, which corresponds to the case when $C_{1} \cap C_{2} \neq \varnothing[8]$.

Example 15 $\mathcal{H}=\mathrm{L}^{2}(\Omega)$, where $\Omega$ is an open bounded domain of $\mathbb{R}^{m}$, and the observed data assume the form $z=L \bar{x}+w$, where $L$ is a bounded linear operator from $\mathcal{H}$ to a Hilbert space $\mathcal{G}$ and $w \in \mathcal{G}$ is an additive noise component. Moreover, $f_{1}: x \mapsto\|L x-z\|_{\mathrm{L}^{1}}$ and $f_{2}=\|\cdot\|_{\mathrm{L}^{1}}$.

\section{ALGORITHM}

Theorem 16 Let $\gamma \in] 0,+\infty\left[\right.$, let $\left(\lambda_{n}\right)_{n \in \mathbb{N}}$ be a sequence in ] $0,2\left[\right.$, and let $\left(a_{n}\right)_{n \in \mathbb{N}}$ and $\left(b_{n}\right)_{n \in \mathbb{N}}$ be sequences in H. Suppose that Problem 11 admits at least one solution, $\sum_{n \in \mathbb{N}} \lambda_{n}\left(2-\lambda_{n}\right)=+\infty$, and $\sum_{n \in \mathbb{N}} \lambda_{n}\left(\left\|a_{n}\right\|+\left\|b_{n}\right\|\right)<$ $+\infty$. Take $x_{0} \in \mathcal{H}$ and set, for every $n \in \mathbb{N}$,

$$
x_{n+\frac{1}{2}}=\operatorname{prox}_{\gamma f_{2}} x_{n}+b_{n}
$$

and

$$
\begin{aligned}
x_{n+1} & =x_{n}+ \\
& \lambda_{n}\left(\operatorname{prox}_{\gamma_{1}}\left(2 x_{n+\frac{1}{2}}-x_{n}\right)+a_{n}-x_{n+\frac{1}{2}}\right) .
\end{aligned}
$$

Then $\left(x_{n}\right)_{n \in \mathbb{N}}$ converges weakly to some point $y \in \mathcal{H}$ and $x=\operatorname{prox}_{\gamma f_{2}}$ x is a solution to Problem 11.

Proof. Let $A$ and $B$ be the subdifferentials of $f_{1}$ and $f_{2}$, respectively, in [1, Corollary 5.2] and use Lemma 2.

In the above algorithm, the minimization problem (9) is decomposed in two main steps: at iteration $n$, the current iterate $x_{n}$ and the function $f_{2}$ is first utilized in (10) to compute $x_{n+\frac{1}{2}}$. The function $f_{1}$ is then utilized to produce the update $x_{n+1}$ in (11). Note that the algorithms allows for the inexact implementation of these two proximal steps via the incorporation of errors $b_{n}$ and $a_{n}$. Moreover, a variable relaxation parameter $\lambda_{n}$ gives added flexibility. It is important to note tha the solution to Problem 11 is obtained as the image under $\operatorname{prox}_{\gamma f_{2}}$ of the weak limit of $\left(x_{n}\right)_{n \in \mathbb{N}}$ and that in general little is known about the asymptotic behavior of $\left(\operatorname{prox}_{\gamma f_{2}} x_{n}\right)_{n \in \mathbb{N}}$ unless $\operatorname{prox}_{\gamma f_{2}}$ is weakly continuous. This is in particular the case when $\operatorname{dim} \mathcal{H}<+\infty$ (by continuity of $\operatorname{prox}_{\gamma f_{2}}$, see Lemma 4(ii)) and we can then formulate a sharper convergence result, which is immadiately relevant in numerical computations with discretized data.

Corollary 17 Suppose that $\operatorname{dim} \mathcal{H}<+\infty$ in Theorem 16. Then $\left(\operatorname{prox}_{\gamma f_{2}} x_{n}\right)_{n \in \mathbb{N}}$ converges (strongly) to a solution to Problem 11.

\section{NUMERICAL RESULTS}

We consider... 


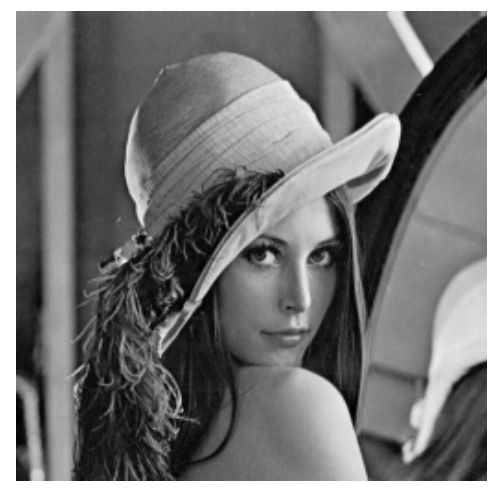

Fig. 1. Original image.

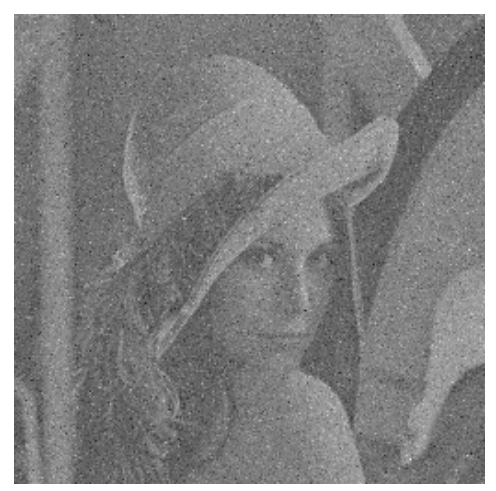

Fig. 2. Noisy image.

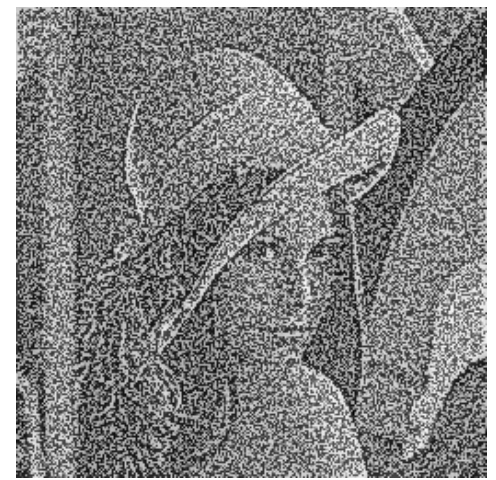

Fig. 3. Denoised image.

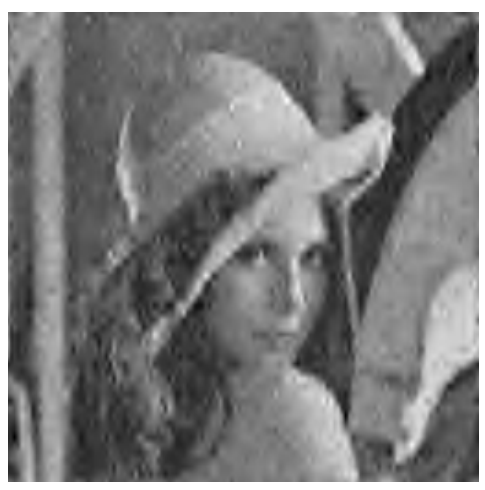

Fig. 4. Denoised image.

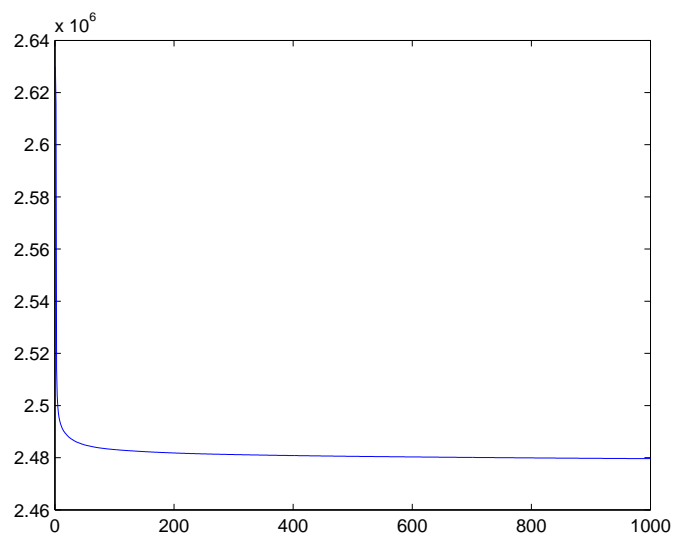

Fig. 5. Convergence profile.

\section{REFERENCES}

[1] P. L. Combettes, "Solving monotone inclusions via compositions of nonexpansive averaged operators," Optimization, vol. 53, pp. 475-504, 2004.

[2] P. L. Combettes and J. C. Pesquet, "Image restoration subject to a total variation constraint," IEEE Trans. Image Proces., vol. 13, pp. 1213-1222, 2004.

[3] P. L. Combettes and V. R. Wajs, "Signal recovery by proximal forward-backward splitting," Multiscale Model. Simul., vol. 4, 2005.

[4] I. Daubechies, M. Defrise, and C. De Mol, "An iterative thresholding algorithm for linear inverse problems with a sparsity constraint," Comm. Pure Appl. Math., vol. 57, pp. 1413-1457, 2004. 
[5] C. de Mol and M. Defrise, "A note on wavelet-based inversion algorithms," Contemp. Math., vol. 313, pp. 85-96, 2002.

[6] D. Dung, "Non-linear approximations using wavelet decompositions," Vietnam J. Math., vol. 29, pp. 197-224, 2001.

[7] P. L. Lions and B. Mercier, "Splitting algorithms for the sum of two nonlinear operators," SIAM J. Numer. Anal., vol. 16, pp. 964-979, 1979.

[8] D. C. Youla and H. Webb, "Image restoration by the method of convex projections: Part 1 - theory," IEEE Trans. Medical Imaging, vol. 1, pp. 81-94, Oct. 1982.

[9] C. Zălinescu, Convex Analysis in General Vector Spaces. River Edge, NJ: World Scientific, 2002.

in long paper let us not forget to discuss Spingarn 